\title{
PRISE EN CHARGE ACTUELLE DES EJACULATIONS RETARDEES ET DES ANEJACULATIONS PSYCHOGENES
}

\author{
Docteur Robert Gellman, Mme Claire Gellman-Barroux
}

\author{
Clinique Urologique de l'Hôpital Necker - 149, rue de Sèvres - 75015 PARIS
}

CURRENT MANAGEMENT OF DELAYED EJACULATION AND PSYCHOGENIC ANEJACULATION. The authors have reviewed their management of 220 patients presenting with retarded ejaculation or psychogenic anejaculation between 1973 and 1992. There are three current management approaches : mechanical treatment (vibrator massage) ; behavioural and cognitive therapy (relaxation, couple therapy) and psychotherapy, indicated when the problem is one of desire. Therapeutic intervention must procede in several stages in the most difficult cases : developing an awareness of subconscious defenses ; rehabilitation and relaxation; and untervention at the couple level. This therapeutic strategy has led to a high success rate of $87 \%$ of cases, although it requires motivated patients and therapists committed to developing relationships of sexual harmony. Key words : retarded ejaculation, psychogenic anejaculation, anorgasmia mechanical treatment, rehabilitation, psychotherapy. Andrologie, 1992, 2 : 134-136

Les éjaculations retardées et les anéjaculations sont des symptômes rares comparativement aux autres symptômes sexuels masculins.

Il s'agit d'un symptôme mystérieux puisque le désir existe, le rapport sexuel est possible mais n'est pas couronné par l'éjaculation comme l'écrivent Masters et Johnson. (4)

C'est un symptôme capricieux, l'absence d'éjaculation pouvant être primaire (le sujet n'a jamais éjaculé) ou secondaire (le sujet a éjaculé au moins une fois dans son existence). C'est un symptôme variable: absence totale d'éjaculation, possibilité d'éjaculation nocturne durant le sommeil, éjaculation possible uniquement par auto masturbation solitaire, plus rarement par auto masturbation en présence de la partenaire ou sous l'effet de caresses ou de fellations.

C'est un symptôme qui peut être sélectif, faisant intervenir le type de la partenaire, les circonstances de la rencontre sexuelle.

Les éjaculateurs retardés sont plus nombreux mais ne consultent guère. Leur symptôme est en effet valorisé dans notre culture car ils peuvent provoquer de multiples orgasmes. C'est seulement si la partenaire se plaint que nous sommes amenés à les voir. Ils réagissent bien aux techniques de relaxation ou aux tranquillisants à doses filées.

C'est essentiellement des anéjaculations que nous aurons à traiter. Nous n'aborderons pas le côté organique qui est très rare, parfaitement explicité dans l'ouvrage de J. Buvat (1) consacré aux troubles de l'éjaculation. Nous nous intéresserons exclusivement à l'aspect psychogène du symptôme.

\section{INTERVENTIONS THERAPEUTIQUES}

Il en existe actuellement 3 types :

- Les moyens mécaniques,

- les interventions de type rééducatif et cognitif, - les interventions psychothérapiques.

\section{1) Moyens mécaniques}

Du fait de leur apparente facilité d'utilisation, l'andrologue serait tenté de les privilégier sans se soucier réellement de la problématique du patient et du couple ni des conséquences possibles de cette intervention.

Dans le cadre du traitement de la stérilité, le but peut être d'arracher une éjaculation au sujet afin de permettre une insémination réclamée par le couple. L'éjaculation est obtenue dans certains cas par chocs électriques : une électrode étant placée dans l'anus et l'autre sur la verge. Ce type d'intervention est emprunté à l'art vétérinaire et se justifie chez certains jeunes paraplégiques dont on veut conserver rapidement un sperme de bonne qualité (avant d'éventuelles complications infectieuses) en vue d'une fécondation ultérieure. Ce type d'intervention mécanique n'entre pas dans notre propos.

L'utilisation du vibromasseur est parfois proposée. Il en existe différents modèles: les plus adaptés possèdent un rhéostat permettant de faire varier l'intensité des vibrations et divers embouts. Il faudra, bien entendu, commencer par les excitations de plus faibles amplitudes et augmenter progressivement.

Avant de prescrire l'usage de ce type d'appareil, il faudrait s'interroger sur la personnalité des patients afin de faire une discrimination entre les troubles fonctionnels et les troubles au niveau du désir.
On retrouve souvent dans leurs antécédents une éducation sévère, rigide, très religieuse. Dans ce cadre, la masturbation n'a jamais été pratiquée ou bien s'accompagnait de scrupules, de remords, de culpabilité.

Les échanges verbaux au niveau de la sexualité n'ont jamais existé au niveau familial. Ils se sont mariés jeunes, sans expérience sexuelle préalable ou à la suite de quelques essais infructueux. Leur partenaire présentait souvent des caractéristiques similaires. Avec cette dernière, ils se montrent très prudes, il y a pas ou très peu de préliminaires à l'acte sexuel. Ils n'arrivent pas à se mettre dans un état d'excitation suffisant au cours du rapport tant ils sont préoccupés de ne pas perdre leur contrôle.

Le but des relations sexuelles est généralement la procréation. C'est d'ailleurs la motivation la plus fréquente de leur demande d'aide thérapeutique.

Une intervention thérapeutique de type uniquement mécanique est rarement suffisante pour résoudre les problèmes sexuels de ces patients.

Cela peut permettre d'obtenir la quantité de sperme suffisante pour des fécondations ultérieures mais le plus souvent ces interventions utilisées seules renforcent les défenses du patient quant à la sexualité et l'éjaculation et n'entraînent pas une sexualité épanouie au niveau du couple.

Il en est de même des alpha stimulants en cours d'expérimentation, utilisés per os ou par voie I.V.

\section{2) Interventions de type rééducatif et cognitif.}

Si le patient est seul à consulter, on préconisera une technique de relaxation pour lui apprendre à se détendre et à se concentrer sur ses sensations.

C'est dans un état de détente corporel qu'on lui demandera de se masturber, en acceptant les fantasmes qui lui viennent spontanément ou qu'il viendrait à se suggérer.

C'est également dans ce contexte que le vibromasseur peut être utilisé en cas d'insuffisance de l'excitation obtenue par la seule automasturbation. Une fois le plaisir orgasmique obtenu de façon régulière, on peut escompter que le sujet cherchera à réitérer cette expérience dans ses relations de couple. 
Actuellement, les patients avertis consultent le plus fréquemment avec leur partenaire: une intervention au niveau du couple se doit alors de leur être proposée.

Nous entendons par là une véritable cothérapie de couple comprenant des entretiens préliminaires croisés avec deux thérapeutes de sexes et de formations différentes et complémentaires, en ménageant ensuite les différentes étapes décrites par Masters et Johnson (4). Le plus souvent on est amené parallèlement à la cothérapie de couple à pratiquer des techniques de relaxation, des entretiens psychothérapiques individuels limités dans le temps, voir un travail sur les fantasmes du sujet.

Le vibromasseur est éventuellement utilisé comme un médiateur à la fois pour des caresses corporelles et pour les caresses génitales. Il est alors considéré comme une aide au traitement et non pas un traitement à lui seul.

\section{3) Interventions psychothérapeutiques}

Parfois les abords pédagogiques et rééducatifs ne paraissent pas appropriés d'emblée du fait de l'importance du contexte névrotique. Le symptôme ne se situe pas au niveau fonctionnel mais au niveau du désir.

Le sujet a profondément refoulé des désirs sexuels condamnés par son sur-moi :

- homosexualité latente,

- pédophilie,

- fétichisme,

- bestialité,

- désirs incestueux,

- désirs sado-masochistes,

- exhibitionnisme, voyeurisme.

Une intervention analytique préalable paraît nécessaire.

Il faudra tenir compte de divers paramètres:

- âge du sujet,

motivations,

- capacités intellectuelles,

- possibilités de verbalisation, d'association libre, - de fantasmatisation, de mémorisation onirique

pour s'orienter vers une technique :

- psychothérapies analytiques brèves, centrées sur le symptôme

- narco-analyse sub-amphétaminée,

- hypnose,

- rêve éveillé dirigé.

Ce n'est que dans les cas les plus problématiques où existent d'autres troubles de la personnalité qu'on orientera le patient vers une psychanalyse classique.

Il faut noter que ces différents modes d'abord agissent au niveau du Moi du sujet, ils n'ont pas pour but premier la levée du symptôme qui n'est guéri que de "surcroit".
Ces techniques permettent dans un second temps d'entreprendre une sexothérapie individuelle ou de couple qui n'aurait pas été possible d'emblée.

\section{EXEMPLES CLINIQUES}

\section{Observation 1}

Monsieur et Madame R. viennent consulter pour non-consommation de mariage après trois ans de vie commune. Au cours des entretiens préliminaires, Monsieur R. nous dit qu'il ne s'est jamais masturbé: "ce n'est pas mon problème" précise-t-il.

Il n'a jamais eu d'éjaculation à l'état de veille, mais en revanche a des pollutions nocturnes. Il n'a eu aucune expérience sexuelle avant son mariage, même avec sa fiancée. Les premières tentatives d'intromission ont été pour lui très douloureuses et se sont heurtées au vaginisme de son épouse, très anxieuse et phobique. $\mathrm{Ce}$ symptôme vaginique, cède assez rapidement avec l'aide de la relaxation et de la prise de conscience des organes génitaux féminins.

On entreprend alors une cothérapie de couple discontinue en s'aidant d'interprétations analytiques comme nous le faisons habituellement.

Nous arrivons rapidement à résoudre le problème de la pénétration. Mais, se révèle alors la véritable difficulté: l'anéjaculation du mari.

Un jour, Monsieur R. arrive à son rendezvous en nous disant qu'il n'a pas pu avoir de rencontre avec son épouse car il souffrait de céphalées violentes, en relation avec une constipation opiniâtre, constipation dont il souffre depuis son adolescence.

- "vous vous retenez encore" interprétons-nous. Il sourit, amusé.

A la séance suivante, nous apprenons que le couple a eu sa première relation sexuelle satisfaisante, avec éjaculation intra vaginale.

- "vous ne vous retenez plus" disons-nous.

Aux questions que nous lui posons sur le vécu de cette première éjaculation, il nous répond avec humour: "je n'ai pas été déçu".

Dans cette observation, la cothérapie de couple a permis de traiter efficacement d'une part l'anéjaculation, mais également la constipation dont Monsieur R. souffrait de façon habituelle depuis de multiples années, par la prise de conscience des mécanismes de rétention propres à sa personnalité.

\section{Observation 2}

Monsieur et Madame L. consultent également pour non consommation de mariage et anéjaculation lors des masturbations et frigidité de l'épouse.

Etant donné l'importance des problèmes névrotiques chez l'un et l'autre partenaire, la cothérapie de couple ne peut être appliquée.

Madame L. entreprend une psychothérapie analytique qui sera suivie d'une psychanalyse, et Monsieur L. une psychanalyse d'emblée.

La sexualité du couple s'améliore progressivement. La pénétration devient possible et agréable. Reste le problème de l'anéjaculation.

Au cours de la cure, le patient parvient à exprimer des fantasmes fétichistes: l'objet de son désir est le pied nu. L'analyse de ce fantasme permettra d'obtenir l'éjaculation intra-vaginale.

La cure psychanalytique doit cependant se poursuivre car les problèmes névrotiques sont loin d'être tous résolus.

\section{Observation 3}

Monsieur V. consulte seul pour anéjaculation intra-coïtale. Il a bénéficié depuis plus de 10 ans, de multiples traitements en relation avec de gros troubles névrotiques (psychanalyse, psychothérapie de soutien, psychodrame, méthodes de groupe, prise en charge pharmacologique). Le seul traitement qu'il n'ait jamais tenté est l'hypnose qu'il réclame à grands cris.

Nous n'entreprenons ce traitement qu'après avoir établi une relation transférentielle positive par une psychothérapie analytique en face à face.

Au cours des séances d'hypnose, il exprime des fantasmes agressifs et d'automutilation.

Nous induisons des fantasmes réparateurs. Le problème de l'anéjaculation se résout alors avec sa partenaire.

Les troubles névrotiques existant par ailleurs nécessitent cependant la poursuite de la psychothérapie analytique. Il faut noter que la résolution du problème sexuel permet l'accélération de la cure.

\section{Observation 4}

Monsieur M. présente d'importantes séquelles d'hémiplégie à la suite de la chute d'un baobab survenue pendant son service militaire à Madagascar. Rééduqué de façon remarquable depuis plusieurs mois, il a récupéré une certaine motricité. Il a par ailleurs noué des relations affectives et sexuelles avec son infirmière qu'il épouse. D'abord impuissant, il a pu grâce à l'aide de cette dernière parvenir à la pénétration, mais il prend alors conscience de son anéjaculation et vient nous consulter.

Etant donné le niveau de la lésion, les neurologues ont rejeté l'hypothèse d'une anéjaculation d'origine organique. L'absence de troubles névrotiques et le caractère très réactionnel de cette anéjaculation nous font entreprendre une relaxation par le Training Autogène de SCHULTZ, dans le but de maîtriser le corps, de le revaloriser sur le plan narcissique, et de parfaire l'intégration du schéma corporel. 
$\mathrm{Au}$ bout de quelques séances, cette anéjaculation fonctionnelle disparait.

\section{RESULTATS}

Nous avions présenté au ler congrès de la Société d'Andrologie, en décembre 1983, une étude portant sur 128 patients ayant consulté pour éjaculation retardée ou anéjaculation psychogène, que nous avions traités au cours des dix précédentes années (2).

Notre statistique concerne maintenant 220 patients ayant accepté de poursuivre un traitement et vus entre 1973 et 1992. La durée moyenne du traitement a été de 5 mois et une semaine et a pu atteindre 15 mois. Durant ces dix dernières années nous avons utilisé de façon quasi systématique des associations de techniques psychothérapiques, corporelles, des traitements de couple avec, dans ce cadre, utilisation éventuelle du vibromasseur.

Cette association de techniques permet de contourner les défenses inconscientes mises en place par les patients et leurs partenaires. Elle nécessite des patients motivés.

Ces conditions réunies entraînent un pourcentage important de résultats positifs, c'est-àdire d'éjaculations intra-vaginales. Sur 220 patients, nous avons obtenu la guérison du symptôme dans $87 \%$ des cas.

$13 \%$ de nos patients ne sont pas parvenus à une éjaculation intra-vaginale mais le symptôme pouvait être considéré comme amélioré :

- possibilité d'éjaculation dans certaines circonstances par automasturbation dans les cas où ils n'y parvenaient pas antérieurement,

- éjaculation par masturbation de la partenaire alors que le tableau initial était celui d'une anéjaculation totale.

\section{COMMENTAIRES ET CONCLUSIONS}

L'anéjaculation psychogène est un symptôme rare et sérieux.

Les premiers sexologues conseillaient de ne pas intervenir au point de vue thérapentique car cette fixation de type anal peut constituer une défense à l'égard d'une structure psychotique sous-jacente. Ils citaient nombre de destructurations graves de la personnalité, de tentatives d'autolyse, d'états dépressifs sévères, de problèmes psycho-somatiques apparus au décours d'un abord direct du symptôme ou lors de sa résolution spontanée.

Si à l'heure actuelle on se montre moins réservé, voir plus interventionniste, on se doit cependant d'être prudent et de n'entreprendre une intervention thérapeutique qu'après avoir apprécié la personnalité du sujet, ses modes de défense et ses motivations réelles à la disparition du symptôme, son caractère fonctionnel ou profond au niveau du désir.

Il ne faut pas oublier que l'anéjaculation psychogène n'est pas une impossibilité physiologique, mais un refus inconscient.

C'est pour cela qu'une intervention thérapeutique comprenant plusieurs étapes nous paraît justifiée dans les cas les plus difficiles.

- prise de conscience des défenses inconscientes,

- temps de rééducation et de détente par la relaxation,

- intervention au niveau du couple dans les cas les plus graves où les symptômes des deux partenaires se confortent mutuellement.

Cette stratégie thérapeutique, utilisant de facon raisonnée l'association de plusieurs techniques complémentaires, nous parait constituer l'apport original de ces dix dernières années dans le traitement de ce symptome difficile.

Dans ce cadre, il faut noter le développement : - des thérapies cognitivo-comportementales, - des thérapies utilisant les fantasmes.

La compréhension psycho-dynamique du symptome nous parait toujours essentielle.

En conclusion, l'éjaculation retardée et l'anéjaculation psychogène nous paraissent nécessiter pour leur prise en charge une stratégie thérapique comportant, selon les cas, une ou plusieurs étapes incluant de façon préférentielle la partenaire, les prescriptions médicamenteuses (stimulants) et le vibromasseur constituent un atout précieux, mais ne peuvent être considérés comme des traitements à eux seuls, si l'on se donne pour objectif une sexualité satisfaisante au niveau du couple et pas uniquement l'obtention d'une éjaculation en vue d'une fécondation ou d'une satisfaction narcissique ponctuelle.

\section{REFERENCES}

1 - Buvat J. Jouannet. L'éjaculation et ses perturbations. Edit. Simep, Villeurbanne, 1984.

2 - Gellman R., Gellman-Barroux Cl. Traitement psychothérapique des anéjaculations psychogènes et des éjaculations retardées. Communication au ler Congrés de la Société d'Andrologie de Langue Française, Lyon, 1983.

3 - Gellman R., Gellman-Barroux cl. Contribution à l'Eude de l'absence d'éjaculation in Sexologie Perspectives Actuelles (sous la direction de Bergeron et Trempe J.P.), Québec, Les Presses de l'Université 1978: 159-163.

4 - Masters et Johnson. Les mésententes sexuelles et leur traitement. Ed. Robert Laffont, Paris, 1974.

RESUME : Les auteurs font état de leurs résultats portant sur le traitement des éjaculations retardées et des anéjaculations psychogènes entre 1973 et 1992 concernant 220 patients. Il existe à l'heure actuelle trois types d'intervention thérapeutique : les moyens mécaniques (vibromasseurs), les traitements rééducatifs et cognitifs (relaxation - thérapies de couple), les traitements psychothérapiques à préconiser lorsque le trouble se situe au niveau du désir. Une intervention thérapeutique comprenant plusieurs étapes est nécessaire dans les cas les plus difficiles : prise de conscience des défenses inconscientes, temps de rééducations et de détente par la relaxation, intervention au niveau du couple. Cette stratégie thérapeutique entraîne un pourcentage important de résultats positifs ( $87 \%$ des cas). Elle nécessite des patients motivés et des thérapeutes soucieux d'obtenir une sexualité harmonieuse au niveau du couple. Mots clés : éjaculations retardées, anéjaculation psychogène, traitements mécaniques, traitements rééducatifs, psychothérapies. Andrologie, 1992, 2 : 134-136. 